\title{
Relativistic effects in the solar EOS
}

\section{A helioseismic forward analysis}

\author{
A. Bonanno ${ }^{1}$, A. L. Murabito ${ }^{2}$, and L. Paternò ${ }^{2}$ \\ 1 Osservatorio Astrofisico di Catania, Città Universitaria, 95123 Catania, Italy \\ 2 Dipartimento di Fisica e Astronomia dell'Università, Sezione Astrofisica, Città Universitaria, \\ 95123 Catania, Italy \\ e-mail: amu@sunct.ct.astro.it; lpaterno@alpha4.ct.astro.it
}

Received 18 April 2001 / Accepted 14 June 2001

\begin{abstract}
We study the sensitivity of the sound speed to relativistic corrections of the equation of state (EOS) in the standard solar model by means of a helioseismic forward analysis. We use the latest GOLF/SOHO data for $\ell=0,1,2,3$ modes to confirm that the inclusion of the relativistic corrections to the adiabatic exponent $\Gamma_{1}$ computed from both OPAL and MHD EOS leads to a more reliable theoretical modelling of the innermost layers of the Sun.
\end{abstract}

Key words. Sun: interior - Sun: oscillations - equation of state

\section{Introduction}

It has recently been shown (Elliott \& Kosovichev 1998) that the inclusion of relativistic effects in the equation of state (EOS) leads to a very good agreement between the solar models and the seismic Sun. In particular, the inversions of SOI-MDI/SOHO $p$-mode frequencies for the adiabatic exponent $\Gamma_{1}$ show that MHD EOS reproduces the interior of the Sun with great accuracy, when the relativistic contribution to the Fermi-Dirac statistics is included.

It is thus interesting to approach the same problem by means of the forward analysis by comparing the theoretical eigenfrequencies with the observed ones. Unfortunately this method is not directly applicable since our description of the outer layers of the Sun is still far from complete and many theoretical uncertainties would influence our conclusions. However, since such small effects in solar EOS are most important only in the deep interior, it is possible to make use of the acoustic mode frequency small separation diagnostic, $\delta \nu_{\ell, n}=\nu_{\ell, n}-\nu_{\ell+2, n-1}$, for spherical harmonic degrees $\ell=0,1$ and radial order $n \gg \ell$ (Tassoul 1980). The main property of this quantity is that it is strongly sensitive to the sound speed gradient near the solar centre while it is weakly dependent on the details of the treatment of the outer layers. Since the relativistic effects manifest themselves mainly through a depletion of $0.1 \%-0.2 \%$

Send offprint requests to: A. Bonanno, e-mail: abo@sunct.ct.astro.it of the adiabatic index $\Gamma_{1}$, we expect a quantitatively similar change of sound speed gradient in the solar core.

The acoustic mode frequency small separation analysis has recently been used for estimating the seismic age of the Sun (Dziembowski et al. 1998) and the related implications of the uncertainties in the $\mathrm{S}_{11}$ astrophysical factor determinations (Bonanno \& Paternò 2001).

Here we show that the mentioned above analysis can also be used to verify how the different physical characteristics of the MHD and OPAL EOS reflect on the accuracy of the description of the stratification of the internal layers of the Sun. On performing a $\chi^{2}$ analysis of the latest published GOLF/SOHO data for different solar models, we confirm the main conclusion of Elliott \& Kosovichev (1998), based on an inversion analysis, that the inclusion of the relativistic effects in the EOS is in any case required to improve the accuracy of solar models, independent of which EOS is used.

\section{The solar model}

In our analysis we used the GARching SOlar Model (GARSOM) code which has been described in detail in Schlattl et al. (1997). It includes the latest OPAL-opacities and either OPAL or MHD EOS, and it takes into account the microscopic diffusion of the elements heavier than hydrogen. Our standard solar model has been verified in detail in Turck-Chièze et al. (1998) and found in good agreement with other up-to-date solar models, and, in particular, it is consistent with the observed $L_{\odot}$ and 
$T_{\text {eff }}$ within $10^{-4}$, at an age of $4.60 \mathrm{~Gy}$, adopting the surface value $Z / X=0.0245$. We then included the relativistic correction leading term to the adiabatic index $\Gamma_{1}$ derived from the relativistic evaluation of the Fermi-Dirac integrals of the EOS in the solar core by means of the expression (Elliott \& Kosovichev 1998):

$\frac{\delta \Gamma_{1}}{\Gamma_{1}} \simeq-\widetilde{T} \frac{2+2 X}{3+5 X}$

where $\widetilde{T}$ is a dimensionless temperature in units of $m_{\mathrm{e}} c^{2} / k$, with $m_{\mathrm{e}}$ the electron mass, $c$ the light speed in vacuum, $k$ the Boltzmann constant, and $X$ the hydrogen abundance by mass. As expected, the correction to $\Gamma_{1}$ is negative, namely $\Gamma_{1, \text { rel }}<\Gamma_{1, n \mathrm{r}}$, since $\Gamma_{1}$ tends to shift from the non-relativistic value of $5 / 3$ to the extremely relativistic one of $4 / 3$.

The corresponding relativistic corrections to the leading terms for sound speed, $c_{\mathrm{s}}$, and density, $\varrho$, are respectively:

$\frac{\delta c_{\mathrm{s}}}{c_{\mathrm{s}}} \simeq \frac{1}{2} \frac{\delta \Gamma_{1}}{\Gamma_{1}}-\frac{15}{64 \sqrt{2}} \widetilde{T} \mathrm{e}^{\psi}$

and

$\frac{\delta \varrho}{\varrho} \simeq \frac{15}{8} \widetilde{T}\left(1+\frac{\mathrm{e}^{\psi}}{4 \sqrt{2}}\right)$

where $\psi$ is the degeneracy parameter, that is about -1.14 at the Sun's centre, and decreases noticeably toward the surface, the partial degeneracy being completely removed at $0.4 R_{\odot}$.

The behaviour, as functions of the fractional radius, of the relative differences between the quantities $\Gamma_{1}, c_{\mathrm{s}}$ and $\varrho$ calculated with relativistic corrections and without them is shown in Fig. 1.

The term $-(15 / 64 \sqrt{2}) \widetilde{T} \mathrm{e}^{\psi}=\delta P / P-\delta \varrho / \varrho$, in Eq. (2) is negligible with respect to $\delta \Gamma_{1} / \Gamma_{1}$ indicating that the relativistic corrections to the pressure, $P$, and density, $\varrho$, cancel each other almost completely and the correction to $c_{\mathrm{S}}$ is entirely dominated by the correction to $\Gamma_{1}$. Also the term $\mathrm{e}^{\psi} / 4 \sqrt{2}$ in Eq. (3) is negligible with respect to unity, indicating that in the solar case the coupling between degeneracy and relativistic effects is weak.

\section{Results with GOLF/SOHO data}

We used the latest GOLF/SOHO data for $\ell=0,1,2,3$ obtained with long time series and by taking into account the asymmetric line profile in data reduction (Thiery et al. 2000). In particular, we determined the acoustic mode small spacing difference $\delta \nu_{\ell, n}$ for $\ell=0,1$ and $n \gg \ell$ for our solar model, and studied the difference $\delta \nu_{i, n, \odot}-\delta \nu_{i, n, \text { model }}$ between data and model. We then constructed the two $\chi^{2}$ indicators (Dziembowski et al. 1998; Schlattl et al. 1997)

$\chi_{i}^{2}=\frac{1}{M-m+1} \sum_{n=m}^{M} \frac{\left(\delta \nu_{i, n, \odot}-\delta \nu_{i, n, \text { model }}\right)^{2}}{\sigma_{i, n}^{2}+\sigma_{2+i, n-1}^{2}}$

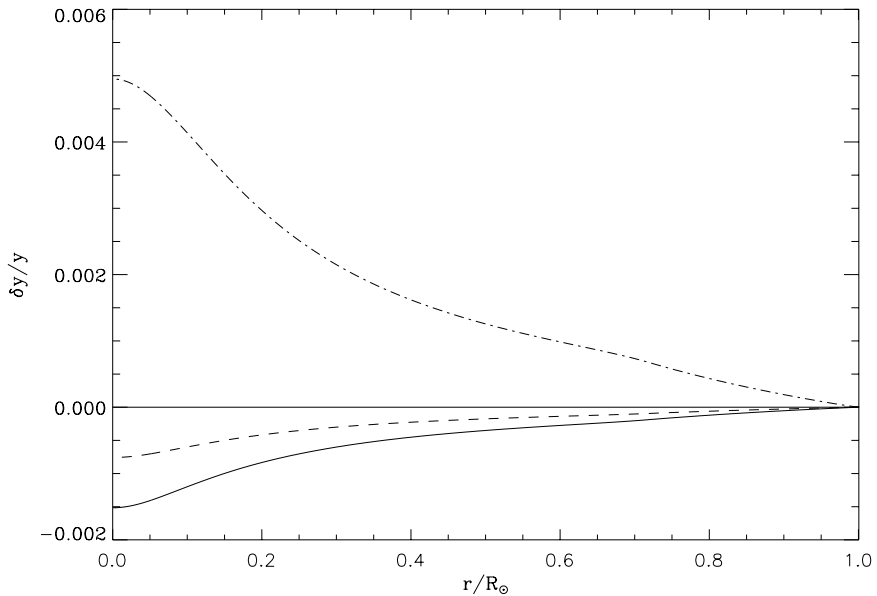

Fig. 1. Behaviour, as functions of the fractional radius, of the relative differences between relativistic and non-relativistic quantities $\delta y / y=\left(y_{\mathrm{rel}}-y_{\mathrm{nr}}\right) / y_{\mathrm{nr}}$, where the $y \mathrm{~s}$ stand for $\Gamma_{1}$ (continuous line), $c_{\mathrm{s}}$ (dashed line), and $\varrho$ (dashed-dotted line) respectively.

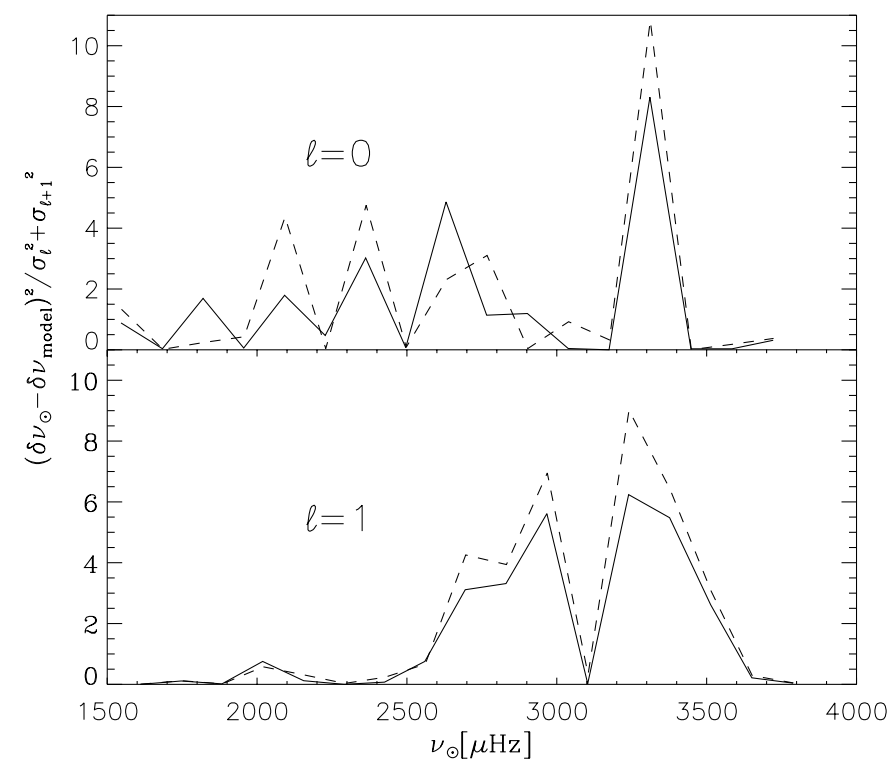

Fig. 2. Relativistic (continuous line) and non-relativistic (dashed line) contribution to the $\chi^{2}$ calculation for MHD EOS.

Table 1. $\chi^{2}$ results in non relativistic (NR) and relativistic (REL) cases for MHD and OPAL EOS.

\begin{tabular}{ccccc}
\hline EOS & $\chi_{0}^{2}(\mathrm{NR})$ & $\chi_{0}^{2}(\mathrm{REL})$ & $\chi_{1}^{2}(\mathrm{NR})$ & $\chi_{1}^{2}(\mathrm{REL})$ \\
\hline MHD & 1.73 & 1.41 & 2.13 & 1.67 \\
$\mathrm{OPAL}$ & 1.91 & 1.41 & 2.32 & 1.94 \\
\hline
\end{tabular}

where $i$ stands for $\ell=0,1, m=10$ and $M=26$. Figures 2 and 3 show the behaviour of the terms in the sum defined in Eq. (4) in non-relativistic and relativistic cases for MHD and OPAL EOS respectively. The difference between the relativistic and non relativistic case is larger for $\ell=0$ in the frequency range beetwen 2000 and $2500 \mu \mathrm{Hz}$, and for $\ell=1$ beetwen 2500 and $3000 \mu \mathrm{Hz}$. The $\chi^{2}$ results are shown in Table 1 where it is possible to note 


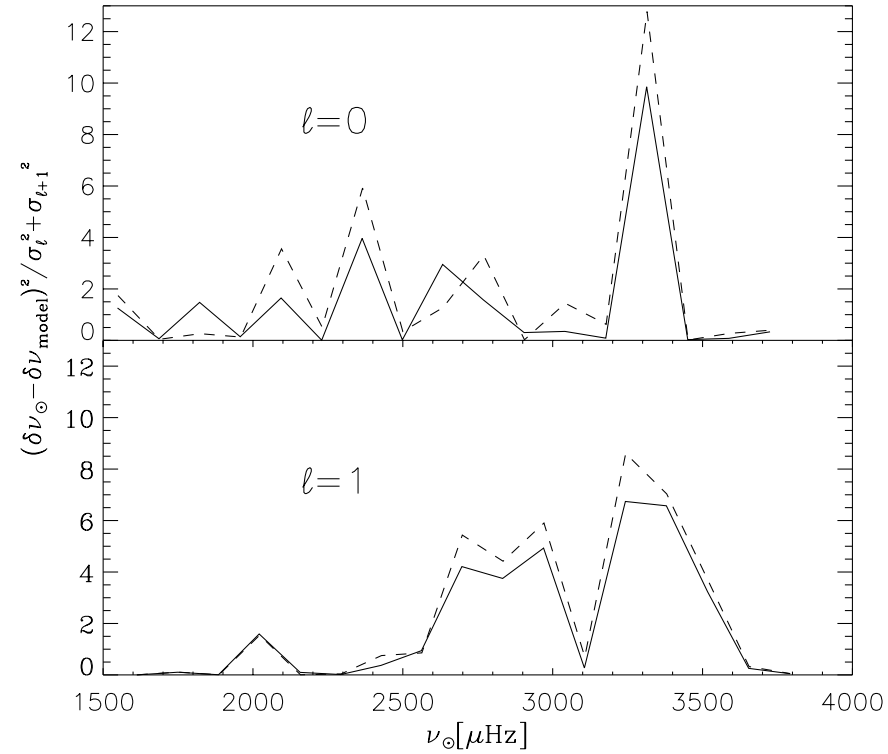

Fig. 3. Relativistic (continuous line) and non-relativistic (dashed line) contribution to the $\chi^{2}$ calculation for OPAL EOS.

that the models with relativistic corrections have rather smaller $\chi^{2} \mathrm{~s}$ and there is no significant difference between $\chi_{0}^{2}$ and $\chi_{1}^{2}$ calculated for OPAL and MHD EOS. However,
MHD EOS appears to be slightly favoured with respect to OPAL EOS.

\section{Conclusions}

Our results show that the acoustic mode frequency small separations are sensitive to the inclusion of the relativistic effects. It would be interesting to discuss the relevance of these effects in the helioseismic determination of the solar age and related problems with $\mathrm{S}_{11}$ uncertainties. We plan to address this issue in a forthcoming communication.

Acknowledgements. We are most grateful to H. Schlattl for useful discussions during the preparation of the manuscript.

\section{References}

Bonanno, A., \& Paternò, L. 2001, Mem. Soc. Astron. Ital., in press

Dziembowski, W. A., Fiorentini, G., Ricci, B., \& Sienkiewicz, R. 1999, A\&A, 343, 990

Elliott, J. R., \& Kosovichev, A. G. 1998, ApJ, 500, L199

Schlattl, H., Weiss, A., \& Ludwig, H. G. 1997, A\&A, 200, L5

Tassoul, M. 1980, ApJS, 43, 469

Thiery, S., Boumier, P., Gabriel, A. H., et al. 2000, A\&A, 355, 743

Turck-Chièze, S., Basu, S., Bertomieu, G., et al. 1998, ESA SP-418, 555 